MASA Fin X:55584

\title{
THE MOTION OF MAGNETIC FIELD LINES
}

\author{
BY \\ DAVID P. STERN
}

GPO PRICE $\boldsymbol{s}$

CFSTI PRICE(S)

JUNE 1966

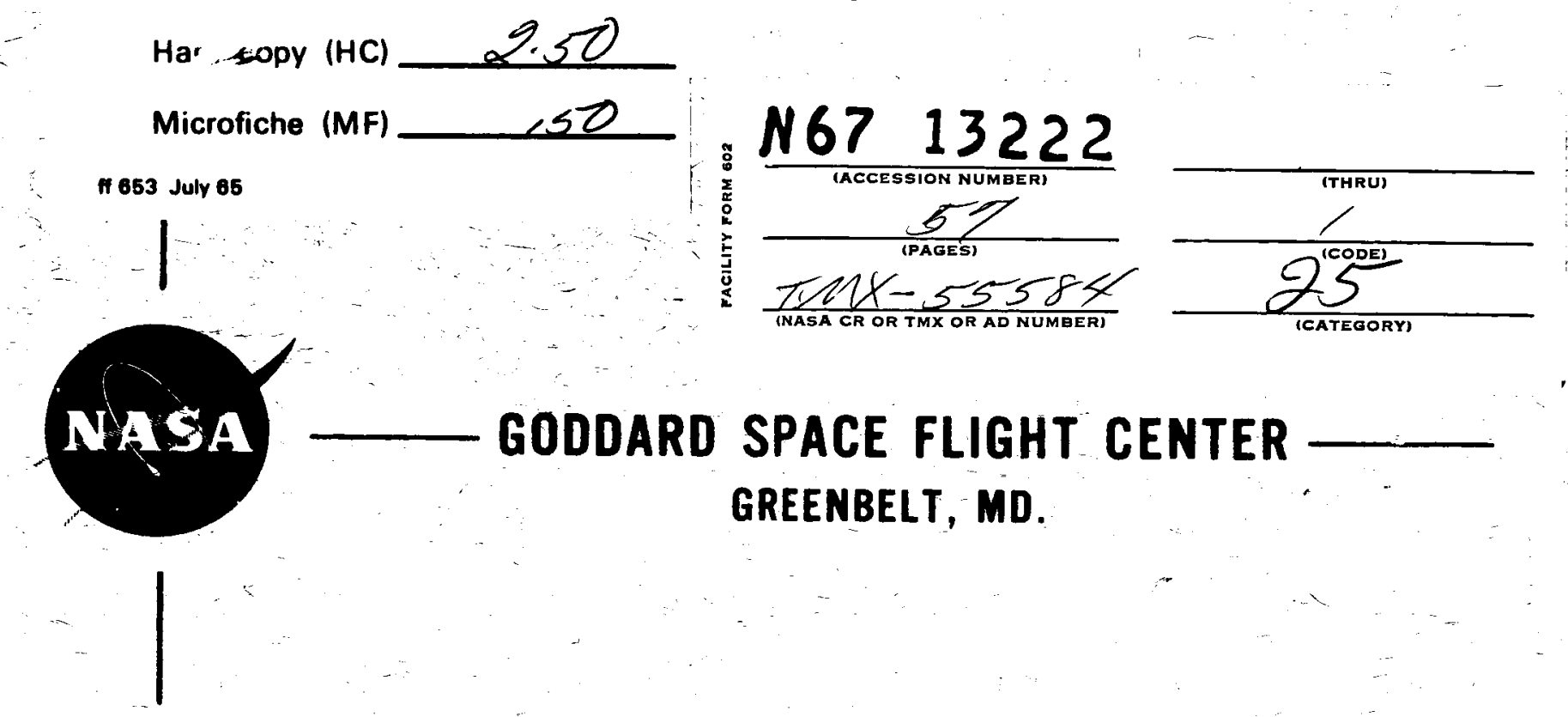


The Motion of Magnetic Field Lines

David P. Stern

Laboratory for Theoretical Studies

Goddard Space Flight Center

Greenbelt, Maryland 20771

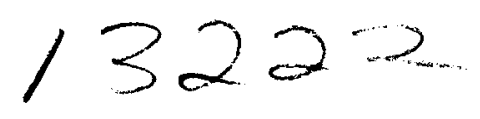

Abstract: The definition and applications of the motion of

magnetic lines of force are reviewed and illustrated.

First, the concept of such a motion is introduced as an

aid to describing the evolution of a known magnetic field.

It is next shown that a known velocity field in a perfectly

conducting fluid with embedded magnetic field may be regarded

as describing the velocity of the magnetic field lines there. The

consequences of such a flow on the field are then derived.

including the properties of flux and line preservation and

Cauchy's general solution. The discussion is then extended to

fluids having a finite high conductivity, with accent on the

role of $x$-type nentral points in the magnetic field. The review

concludes with a brief description of existing theories on the

behavior of such nentral points. 


\section{(1) Introduction}

Every theory in the exact sciences is based on a mathematical formalism which derives its results from some given fundamental relations, In addition, however, it is often possible to formulate statements of a qualitative nature or, at least, with some intuitive content, about the subject described by the theory. Such statements are very useful, for they give insight into the subject's behavior and allow the deduction of general results without excessive calculation.

The statement "in highly conductive fluids, magnetic lines of force move with the material" belongs to this class. In complicated geophysical and astrophysical situations this is often the first guide to the evolution of the system being investigated, from which one may continue, say, by devising simple (e.g. two-dimensional) mathematical models.

Unfortunately, the concept of the motion of field lines has at times caused misunderstanding among those not familiar with its precise meaning and formalistic background. The purpose of this article is to review this meaning and backgronnd and to describe the limitations of the concept and some of its past applications. It is hoped that by following the exposition the reader will gradnally become familiar with the subject and acquire the "intuitive feeling" for it on which much of its application depends.

A great part of the theory of motion of field lines parallels earlier work on vortex lines in ideal fluids. This follows from 
the basic equation for the development of the vorticity $\Omega$ in such fluids

$$
\frac{\partial \rho}{\partial t}-\nabla \times(\underline{v} \times \Omega)=0
$$

which is formally identical with the equation governing the development of the magnetic field $\underline{B}$ in perfectly conducting fluids

$$
\frac{\partial \underline{B}}{\lambda t}-\nabla \times(\underline{v} \times \underline{B})=0
$$

The latter equation is readily obtained by noting that the first order electric field $\underline{E}^{*}$ in the frame of the moving fluid is

$$
\underline{E} *=\underline{E}+(\underline{v} \times \underline{B})
$$

and that Ohm's law then is written

$$
\underline{j} * \sigma E *
$$

Taking the curl of $(1-4)$ and applying Maxwell's equations yields equation $(1-2)$, in the limit as $\sigma \rightarrow \infty$, j* $f^{\infty}$.

An early use of the concept of perfect conductors is due to Maxwel1 (1873; art. 654) who showed that a perfectly conducting closed shell shields its interior from magnetic variations arising outside it. Thus when the conductivity of any material tends to infinity, the magnetic flux existing in it becomes "frozen in" and ceases to be influenced by external fields. Sitch ideas of "frozen flux" were expressed by early theories of superconductivity 
which, however, did not consider a fluid medium and which were abandoned after the discovery in 1933 of the Meissner effect. The viewpoint that in astrophysics ionized gases can often be regarded as highly conducting fluids in which "the liquid is 'fastened' to the lines of force" was first advanced by Alfvén (1942-b) who also used the expression that in this case the lines of force are "frozen-in" into the fluid (Alfvén, 1942-a). The extension of the theorem of conservation of vorticity, derived by Helmholtz in 1858 (and independently, by Dirichlet) to the case of hydromagnetic flow, probably occured at about that time, for Walén in his work on solar magnetism (1946, eq. 12) refers to it as "well known". The fundamental vorticity equation of Cauchy (1815) was similarly translated into hydromagnetic terms by Lundquist and Cowling (Lundquist, $1951,1952)$ and many of the remaining details of the analogy were worked out be Malersberger $(1964)$. In the present work, hydromagnetic equations which originated in the study of vorticity will be referred to by their original names.

The theory described here deals with high ly conducting fluids; most of its applications, however, are to plasmas. In order to justify the extension of $(1-2)$ to plasmas, two extreme situations will be considered. If the plasma is dense and dominated by collisions, fluid equations may be derived. Ohm's law is then considerably more involved than $(1-4)$ (Spitzer, 1962 eq. 2-21), but with appropriate approximations only the pressure $\boldsymbol{\nabla}_{\mathrm{i}}$ (subscript $\mathrm{i}$ for ions) and the gravitational field $\boldsymbol{\nabla} \phi$ have to 
be taken into account (Spitzer, 1962).

$$
\underline{j}=\sigma\left(E^{*}-\frac{1}{n_{e} e} \nabla_{p}-\frac{m_{1}}{Z e} \nabla_{\phi}\right)
$$

If the electron density $\mathrm{n}_{e}$ is approximately constant or if $\mathrm{p}_{i}$ can be regarded as a function of it alone, the additional terms disappear when the curl is taken and $(1-2)$ is obtained as before. In strong magnetic fields o may be anisotropic, but as long as the current flows "easily" (in the sense of eq. 4-3) in any given direction, the high-conductivity approximation can be expected to hold.

At the other extreme one finds the "guiding center fluid" in which collisions are extremely rare. It may be shown, however (Parker, 1957a; Thompson, 1962, ch. 8, \$7) that if the magnetic field is sufficiently strong to maintain the guiding center approximation, the electric field $E^{*}$ of eq. (1-3) vanishes in this case to lowest order in the ratio between the gyration time and the time scale of the macroscopic motions. The basic equation ( $1-2)$ then follows, so that the guiding center fluid may be regarded as a nearly perfect conductor in this connection. It should be added, also, that the slippage of lines of force in a dense plasma due to finite conductivity is much more readily analyzed than that occurring near the fringes of the magnetic field in a guiding-center plasma, in a region where the guiding center approximation becomes inaccurate.

The motions discussed here will be relatively slow ones. Nonrelativistic transformations (e.g. equation 1-3) will be nsed, 
the displacement crrrent will always be neglected and wave phenomena will not be included.

\section{(2) Euler Potentials}

The magnetic field is solenoidal and therefore can be represented by two scalars. This representation may be achieved in several ways; Eor studying magnetic lines of force it is advantageous to introduce Euler potentials (Evler, 1770, $\$ 26$, $\$ 49$; Truesde11, 1954, \$13) nsually denoted by $a$ and $\beta$, with the property

$$
\underline{B}=\nabla_{C} \times \nabla_{\beta}=\nabla \times\left(\alpha \nabla_{\beta}\right)
$$

By this definition $\underline{B}$ is tangent to each of the families of sirfaces $\alpha=\underline{\text { constant }}$ and $=$ constant. The line of intersection of any two such surfaces therefore defines a line of force characterized by the associated values of $\alpha$ and $\beta$. Other commonly used methods of representing $\underline{B}$ afford no such direct way of evaluating lines of force.

The use of Enler potentials also facilitates the introduction of tubes of flux. Consider a flux tube with a rhomboidal cross section, defined by the 4 lines of force $(\alpha, \beta),(\alpha+\mathrm{d} \alpha, \beta)$, $(\alpha, \beta+d \beta)$ and $\left(\alpha+d_{\alpha}, \beta+d \beta\right)$. The flux enclosed by the tribe, evaluated in any plane normal to $\underline{B}$, is

$$
\mathrm{d} \Phi=\int \underline{\mathrm{B}} \cdot \mathrm{d} \underline{\mathrm{s}}=\oint a \nabla_{\beta} \cdot \mathrm{d} \underline{\hat{x}}=\oint \alpha \mathrm{d} \beta
$$

On two sides of the rhomboid $\beta$ is constant and no contribution to the integral arises, while the two remaining ones contribute 
$\left(\alpha+d_{\alpha}\right) d \beta$ and $-\left(\alpha d_{\beta}\right)$. This

$$
\mathrm{d} \Phi=\mathrm{d} \alpha \mathrm{d} \beta
$$

If $\alpha$ and $\beta$ are supplemented by a third spatial coordinate $\gamma$, the flux through an area $S$ on a surface $y=$ constant is then

$$
\Phi=\int_{S} \mathrm{~d} \alpha \mathrm{d} \beta
$$

In static fields, the magnetostatic potential may be chosen as $y$. It should be noted however, that in general it is not possible to stipulate that $y$ varies only along the line of force, i.e. that $\nabla_{\gamma}$ is normal to $\nabla_{\alpha}$ and $\nabla_{\beta}$. Such a stipulation implies $\underline{B}=\varepsilon \nabla \gamma(\epsilon$ some scalar function of position), from which $\underline{B} \cdot(\nabla \times \underline{B})=0$, a condition which is not in general met. The Euler potentials $\alpha$ and $\beta$ each satisfy the first order partial differential equation

$$
\underline{B} \cdot \nabla f=0
$$

and the possibility of expressing $\underline{B}$ by them may be proved (e.g. Phillips, 1933; $\$ 20)$ by using the existence proofs for solutions of this equation. The potentials are not uniquely defined, however: any independent pair of solutions to (2-6) leads to some choice of $\alpha$ and $\beta$. Given a pair of such potentials, an alternate choice (e.g. Ray, 1963) is $h(\alpha, \beta)$ and $g(\alpha, \beta)$, provided

$$
\frac{\partial(g, h)}{\partial(\alpha, \beta)}=1
$$


Euler potentials have two major disadvantages. First of all, the representation is not linear and therefore the superposition of Euler potentials due to several sources is not in general valid. As a result, it is not possible to derive the finctions $\alpha$ and $\beta$ analytically, except in some simple field configurations. One such case is provided by axisymmetric poloidal fields, such as that of a magnetic dipole. The vector potential A may then be taken in the azimuthal direction, leading to the choice

$$
\begin{aligned}
& B=\phi \\
& \underline{A}=\frac{\alpha(r, \theta)}{r \sin \theta} \underline{i} \theta
\end{aligned}
$$

In fluid dynamics eq. $(2-8)$ is often used to describe an axisymmetric fluid velocity field, with $(-a)$ called the Stokes stream function (Milne-Thomson 1960, Chapt. 15; 0'Brien, 1963). Superposition of potentials exists in this case, all of them sharing the same $\beta$. Another case for which a can be derived analytically (and superposed) is provided by purely toroidal fields

$$
\underline{B}=\nabla \times \underline{r} \psi=\nabla \psi \times \nabla \frac{1}{2} r^{2}
$$

Still another is that of a two-dimensional field, where $\underline{B}$ reither depends on $z$ nor has a component in its direction. One may then choose

$$
\underline{B}=\nabla \times A i_{\underline{z}}=\nabla A(x, y) \times \nabla z
$$


The second and more serious disadvantage of Euler potentials is that in most magnetic fields lines of force are not closed and therefore attain infinite length in a bounded region of space. Consider (McDonald, 1954) a current-bearing circle $r=R$ in the $z=0$ plane of a cylindrical coordinate system. Because of axial symmetry, the a surfaces are toroidal rings and the $\beta$ surfaces meridional planes in which the lines of force, which are closed,lie. Now let a current filament be added along the $z$ axis, giving the lines of force a slant and thus transforming them into helices. It may be shown that the a derived without the current filament may still be used; however, a $\beta$ surface now resembles an archimedean screw with its axis twisted around the circle and its ends, in general, not meeting. Except for those cases in which a line of force returns into itself after a finite length (there is an infinite number of such cases, but of measure zero), a line of force will range all over its $a$-surface, with different sections coming arbitrarily close to each other but never meeting.

Given a point on the $\alpha$-surface it is possible to construct a B-surface through it. This surface, however, if continued for a sufficient length, will fill a region in space and approach arbitrarily close to any given point in it. It is then impossible to construct a family of $\beta$-surfaces continuously dependent on a parameter. The characterization of lines of force by families of Euler potentials is therefore not feasible when such lines are bounded but of infinite length.

The difficulty may be lifted by introducing a surface of 
discontinuity which artificially terminates the $(\alpha, \beta)$ lines (Dungey, 1958; §3.1). In the example given the part of the plane $z=0$ with $r \ll R$ may be chosen as such a surface, with each pair of parameters $(\alpha, \beta)$ defining a segment of a line of force from the point where it leaves the top of the plane to where it reaches the bottom. One may still consider infinitely long lines of force but such a line is then characterized by an infinite number of $\beta$ values, with $\beta$ changing every time the line crosses the surface of discontinuity.

\section{(3) Kinematics of Lines of Force}

The first problem associated with motion of lines of force may be posed as follows. Given a magnetic field which changes in time in a known way, can an alternative description of this time-dependence be made by assigning a velocity to its lines of force?

In classical fluid dynamics, the rate of change in some quantity $\lambda$, as observed by a particle moving with the fluid's velocity $\underline{u}_{2}$ is its "material derivative"

$$
\frac{\mathrm{d} \lambda}{\mathrm{d} t}=\frac{\partial \lambda}{\partial t}+\underline{u} \cdot \nabla \lambda
$$

If $\lambda$ is convected with the flow, the material derivative vanishes. It therefore seems reasonable to call $\underline{u}$ the velocity of the lines of force if it satisfies

$$
\begin{aligned}
& \frac{\partial \theta}{\partial t}+\underline{u} \cdot \nabla_{\alpha}=0 \\
& \frac{\partial \beta}{\partial t}+\underline{u} \cdot \nabla \beta=0
\end{aligned}
$$


A velocity satisfying the preceding condition can always be found (Northrop, 1963), namely

$$
\underline{u}=\left(\frac{\partial \beta}{\partial t} \nabla \alpha-\frac{\partial \alpha}{\partial t} \nabla_{B}\right) \times \frac{B}{B^{2}}
$$

as may be verified by substitution. This solution is not unique: any velocity parallel to $\underline{B}$ may be added to it without changing the validity of $(3-2)$, since $\underline{B}$ is normal to both $\nabla \alpha$ and VB. Because the component of $\underline{u}$ parallel to $\underline{B}$ is arbitrary, it is customary to set it equal to zero; when discussing the velocity of a line of force, then, only the component normal to $\underline{B}$ is implied.

This definition $(3-2)$ for the velocity of the lines of force is not entirely satisfactory: the Euler potentials are not directly observable and it is not a-priori certain that equations (3-2) have to be satisfied. Indeed, since at any time there exist many equivalent choices of $\alpha$ and $\beta$ describing the same $\underline{B}$, one may always add to the velocity satisfying (3-2) another velocity which describes a time-dependent transformation of Euler potentials, without altering the physical picture. Such a velocity will be termed a "relabeling velocity". For instance, in an axisymmetric field one can add any (not necessarily steady) angular velocity around the symmetry axis, for such a velocity merely relabels the lines and has no observable effect.

Following Newcomb (1958) we therefore broaden the definition and regard as a "velocity of lines of force" any velocity $\underline{v}$ 
satisfying eq. $(1-2)$

$$
\frac{\partial \underline{B}}{\partial t}-\nabla \times(\underline{v} \times \underline{B})=0
$$

It may be verified that $\underline{u}$ of eq. $(3-3)$ is one of the solutions. Newcomb showed that by this definition all "verifiable con sequences" which are usually associated with the concept of the motion of lines of force are satisfied: the two most important of these consequences, namely line preservation and flux pre.. servation, will be discussed separately in Section 4.

The most general solution of $(1-2)$ will be the sum of the particular solution $(3-3)$ and the general solution $\underline{w}$ of

$$
\boldsymbol{\nabla} \times(\underline{w} \times \underline{B})=0
$$

If one specifies, as before, $(\underline{w} \cdot \underline{B})=0$, this general solution has the form

$$
\underline{w}=\frac{\nabla \Phi \times \underline{B}}{B^{2}}
$$

where $\psi(\alpha, \beta)$ is a scalar conserved along a line of force. Since for any "relabeling velocity" which expresses no variation in the field, equation $(1-2)$ reduces to $(3-4)$, it is clear that such a velocity will have the form $(3-5)$.

(4) The Motion of Perfectly Conducting Fluids

In a conducting fluid the presence of an electric field causes current to flow. If the fluid is moving, the relevant electric field is the one taken in a frame of reference moving 
with the fluid; to first order this field is given by eq. (1-3)

$$
\underline{E}^{*}=\underline{E}+(\underline{v} \times \underline{B})
$$

In the simplest case the relation between $\underline{E}^{*}$ and the current density $j^{*}$ in the moving frame follows Ohm's law and is linear and isotropic as in $(1-4)$

$$
\underline{j}^{*}=\sigma \underline{E} *
$$

For ionized gases in a magnetic field the relation may be anisotropic (the conductivity $\sigma$ is a tensor) or even nonlinear, but in general $\underline{j}^{*}$ and $\underline{E}^{*}$ increase together. For simplicity, a simple ohmic relationship with scalar $\sigma$ will be assumed here, as well as those approximations valid when $v \ll c$. By Maxwell's equations

$$
\frac{\partial \underline{B}}{\partial t}-\nabla \times(\underline{v} \times \underline{B})=\nabla \times \frac{1}{\mu_{0} \sigma}(\nabla \times \underline{B})
$$

or, if $\sigma$ is constant everywhere

$$
\frac{\partial \underline{B}}{\partial t}-\nabla \times(\underline{v} \times \underline{B})=\frac{1}{\mu_{0} \sigma} \nabla^{2} \underline{B}
$$

The case of interest here occurs when $\sigma$ is very large, so that the term involving it may be neglected and (4-1) reduces to $(1-2)$. A rough but useful criterion to indicate when such an omission is permissible is furnished by the magnetic Reynolds number (Elsasser 1955, 1956, 1957). If L is a typical dimension over which $B$ varies and $V$ is a typical velocity, the relative 
magnitude of the terms in (4-3) may be estimated by replacing $\nabla$ with $\mathrm{L}^{-i}$ and $\underline{v}$ by $V$; vector character is neglected in this crude approach. It then follows that the right hand term is negligible only if the dimensionless "magnetic Reynolds number"

$$
\mathrm{R}_{\mathrm{m}}=\mu_{\mathrm{o}} \sigma \mathrm{LV}
$$

is much larger than unity. It is important to note that increasing the scale of the flow increases $R_{m}$; this is why conditions of "perfect conduction" occur much more readily on astrophysical and geophysical scales than in the laboratory.

The discussinn in this work will be mainly concerned with the limiting case $R_{m} \rightarrow \infty$, for it is then that the fluid velocity satisfies the criterion $(1-2)$ for the velocity of the lines of force. In ordinary hydrodynamics, flows with large Reynolds numbers are unstable and tend to break up into turbulent eddies. While turbulence may also occur in the flow of conducting fluids and of plasmas, such behavior will not be considered here since relatively little has been established about this subject to date (see Batchelor, 1950)。

The most important properties of $\underline{B}$ following eq. $(1-2)$ are flux preservation and line preservation.

A flow $\underline{v}$ is said to be flux preserving for a vector field $Q$ if the flux of $Q$ through a surface defined by a closed filament of fluid particles is conserved. It will now be shown that for a 
given magnetic field $\underline{B}$ a flow $\underline{v}$ is flux preserving if $(1-2)$ is satisfied.

Let a fluid filament initially following the closed contour $S$ be given and let $\Phi$ be the initial flux of $\underline{B}$ through it. A short interval dt later, each element $\underline{d \ell}$ of the contour will have been $d i s p l a c e d$ by an amount $\underline{v} d t$, sweeping in the process an area $(\underline{v} \times \underline{d l}) d t(F i g u r e ~ 1)$. In this time interval, $\Phi$ changes by an amount $\mathrm{d} \Phi$, ascribable to two callses. The time variation of the field contributes the surface intergral

$$
\int_{S} \frac{\partial \underline{B}}{\partial t} \cdot d \underline{A} d t
$$

while the variation of the area bounded by the filament adds the flux through the area swept by it (figure l), equaling

$$
\oint \underline{B} \times(\underline{v} \times \underline{d} \ell) d t=-\int \nabla \times(\underline{v} \times \underline{B}) \times d \underline{A} d t
$$

Thus

$$
\mathrm{d} \Phi=\int\left(\frac{\partial \underline{B}}{\partial t}-\nabla \times(\underline{v} \times \underline{B})\right) \times d \underline{A} d t
$$

and this vanishes if $(1-2)$ holds. Because the contour is arbitrary, (1-2) is not only sufficient but also necessary for flux preservation. For a nonsolenoidal vector field $Q$ the condition for flux preservation becomes

$$
\frac{\partial \underline{Q}}{\partial t}-\nabla \times(\underline{v} \times \underline{Q})+\underline{v}(\underline{\nabla} \cdot \underline{Q})=0
$$


A motion is called 1 ine preserving if two fluid particles initially connected by a line of force continue to be so

throughout their motion. A flux-preserving motion is always line preserving, by the following argument (for an alternative derivation, see Newcomb, 1958).

Consider a sheet $A B C D$ of fluid particles which forms part of a magnetic flux trube (Figure 2), with points $A$ and $B$ chosen so as to lie, at some initial instant, on the same line of force. The magnetic flux throngh the sheet and through any closed contonr on it then vanishes, and by flux preservation this property is maintained as time progresses. The sheet will therefore always be part of a flux tube.

The same arguments hold for a sheet $A B C^{\prime} D^{\prime}$ on another flux tube which initially intersects the first one along the field line $\mathrm{AB}$. As time passes, $\mathrm{A}$ and $\mathrm{B}$ will continue to belong to both flux tubes and therefore lie on their intersection, which defines a line of force. Since the choice of $A$ and $B$ is in no other way restricted, the motion is line preserving.

The condition for line preservation is less strict than that for flux preservation. By the Helmboltz-Zorawsi criterion (Zorawski, 1900; for further references see Truesde11, 1954; Truesde11 and Toupin, 1960; al so Newcomb, 1958, eq. 85) a necessary and sufficient condition for line preservation is

$$
\left[\frac{\partial Q}{\partial t}-\nabla \times(\underline{v} \times \underline{Q})+\underline{v}(\nabla \circ Q)\right] \times \underline{Q}=0
$$

The above demonstrates that flux preservation is not necessary 
for line preservation; for instance, motion in a force-free field with finite, constant resistivity is line preserving but not flux preserving. The following proof is due to Prim and Truesdell (1950). Let the equation of an open fluid filament at time $t$, given in terms of some parameter $s$ (e.g. the arc distance from a given particle in the filament) be

$$
\underline{x}=\underline{x}(s, t)
$$

Then $\mathrm{d} \underline{\mathrm{x}} / \mathrm{ds}$ is tangent to the filament and the condition for line preservation is that if initially

$$
\frac{\partial \underline{x}}{\partial s} \times \underline{Q}=0
$$

so that the filament is aligned with a field line of $Q$, then this condition is met at all times. The time variation of the quantity under consideration is

$$
\begin{aligned}
\frac{d}{d t}\left(\frac{\partial \underline{x}}{\partial s} \times \underline{Q}\right) & =\left(\begin{array}{lll}
\frac{\partial \underline{v}}{\partial s} & \underline{Q}
\end{array}\right)+\left(\frac{\partial \underline{\underline{x}}}{\partial s} \times \frac{d Q}{d t}\right) \\
& =\left(\frac{\partial \underline{\underline{x}}}{\partial s} \cdot \nabla \underline{v}\right) \times \underline{Q}+\left(\frac{\partial \underline{x}}{\partial s} \times \frac{d Q}{d t}\right)
\end{aligned}
$$

Now $(4-10)$ is equivalent to

$$
\frac{\partial \underline{\underline{x}}}{\partial s}=\lambda \underline{Q}
$$

If $(4-8)$ holds and $(4-10)$ is initially given, one gets for the initial variation of the expression on the left of $(4-10)$ 


$$
\begin{aligned}
\frac{\mathrm{d}}{\mathrm{dt}}\left(\frac{\partial \mathrm{x}}{\partial \mathrm{s}} \times \underline{Q}\right) & =\lambda \underline{Q} \times\left(\frac{\mathrm{d} \underline{\mathrm{d}}}{\mathrm{dt}}-\underline{Q} \cdot \nabla \underline{\mathrm{v}}\right) \\
& =\lambda \underline{Q} \times\left(\frac{\mathrm{d}-13)}{\mathrm{d} \underline{\mathrm{t}}}-\nabla \times(\underline{\mathrm{v}} \times \underline{Q})+\underline{\mathrm{v}}(\nabla \cdot \mathrm{Q})\right)=0
\end{aligned}
$$

and therefore $(4-10)$ holds for later times as well.

\section{(5) Canchy"s Integral}

Equation (1-2) for the motion of a perfectly conducting fluid involves both $\underline{B}$ and $\underline{v}$. For those cases in which both quantities have to be derived theoretically another equation involving both is required, as well as a set of appropriate boundary conditions. The additional equation is the hydromagnetic generalization of the Navier-Stokes formula and the solution of the combined set, even in simple cases, is a for. midable task。

In practice, however, $\underline{v}$ may often be obtained separately, as for example, when it may be deduced from observations (e.g. the differential rotation of the $\sin$ ) or when magnetic forces are negligible in comparison with others acting on the fluid, in which case the hydromagnetic equation may be separately solved for $\underline{v}$. In such cases one may regard $\underline{v}$ in eq. $(1-2)$ as given and seek a solution for $\underline{B}$.

As was noted before, eq. $(1 \cdot 2)$ was first studied with $\underline{\Omega}$ replacing $\underline{B}$, in which case it describes the development of vorticity in an ideal fluid. It was in this context that its general solution was achieved by Cauchy (1815), who transformed 
the flux-conservation of $\Omega$ into the material conservation of a related quantity.

Equation (1-2) may be rewritten

$$
\frac{\mathrm{d} \underline{\mathrm{B}}}{\mathrm{dt}}=\frac{\partial \underline{\mathrm{B}}}{\partial \mathrm{t}}+\underline{\mathrm{v}} \cdot \nabla \underline{\mathrm{B}}=\underline{\mathrm{B}} \cdot \nabla \underline{\mathrm{v}}-\underline{\mathrm{B}}(\nabla \cdot \underline{\mathrm{v}})
$$

Substituting the equation of continuity

$$
\frac{d \rho}{d t}+\rho(\nabla \cdot \underline{v})=0
$$

gives, after several steps

$$
\frac{\mathrm{d}}{\mathrm{d} t}\left(\frac{\mathrm{B}}{\rho}\right)-\frac{\mathrm{B}}{\rho} \cdot \nabla \underline{\mathrm{v}}=0
$$

which is known (when $\underline{B}$ is replaced by $\Omega$ ) as Helmholtz's equation. Its form suggests a search for an integrating factor $\subseteq$ (of yet undetermined tensorial character) reducing the equation to the form

$$
\frac{d}{d t}\left(\frac{B}{\rho} \cdot c\right)=0
$$

which immediately integrates to

$$
\frac{\underline{B}}{\rho} \cdot \underline{c}=\frac{\underline{B}^{(0)}}{\rho(0)} \cdot \underline{c}^{(0)}
$$

In order to express the integrating factor, material coordinates (sometimes called Lagrangian coordinates, though their use is due to Euler; see Truesdell 1954, footnote in $\$ 14$ ) 
have to be introduced. Its derivation below follows Elsasser (1956); some other derivations may be found in the works of Brand $(1947 ; \S 123)$, Lamb $(1879 ; \S 146)$, Goldstein (1960; $\$ 4.2,4.3)$, Truesdell (1954) and Truesde1l and Toupin (1960). Most of these describe the development of fluid vorticity; the first application to magnetic fields in perfectly conducting fluids is due to Lundquist and Cowling. (Lundquist 1951, 1952). The material description of fluid flow involves referring variables to their values at some initial instant $t=0$; such initial values will be distinguished by superscript (0). Consider an arbitrary fluid particle; in an orthogonal cartesian reference frame its position vector $\underline{x}$ at any time $t$ is uniquely determined by its initial value $\underline{x}^{(0)}$ and by $t$

$$
\underline{x}=\underline{x}\left(x^{(0)}, t\right)
$$

The Jacobian $\mathrm{J}$ of this transformation between $\underline{x}^{(0)}$ and $\underline{x}$ ( $t$ being assumed given) is the ratio, by which a volume element moving with the fluid has changed from its initial value, and therefore, the ratio between initial and final densities in the particle's vicinity

$$
J=\frac{\partial\left(x_{1}, x_{2}, x_{3}\right)}{\partial\left(x_{1}(0), x_{2}(0), x_{3}(0)\right)}=\frac{\rho(0)}{\rho}
$$

Consequently, as long as this ratio is finite, a mique inverse transformation exists

$$
\begin{array}{r}
\underline{x}^{(0)}=\underline{x}^{(0)}(\underline{x}, t) \\
-19 .
\end{array}
$$


The two transformations are related, for any given $t$, by

$$
\left.\frac{\partial x_{i}}{\partial x_{k}}\right)^{-} \cdot \frac{\partial x_{k}}{\partial x_{1}}=\delta_{i 1}
$$

(in the last equation and in those following the summation convention for repeated indices is used).

From this, whether $i$ equals 1 or not,

$$
\frac{d}{d t}\left(\frac{\partial x_{i}}{\partial x_{k}^{(o)}} \frac{\partial x_{k}}{\partial x_{1}}\right)=0=\frac{\partial v_{i}}{\partial x_{k}}+\frac{\partial x^{i}}{\partial x_{k}} \frac{d}{d t}\left(\frac{\partial x_{k}^{(o)}}{\partial x_{1}}\right)
$$

Writing (5-3) in component form and substituting (5-10) gives

$$
\frac{d}{d t}\left(\frac{B_{i}}{\rho}\right)+\frac{B_{1}}{\rho} \frac{\partial x^{i}}{\partial x_{k}^{(o)}} \frac{d}{d t}\left(\frac{\partial x_{k}^{(o)}}{\partial x_{1}}\right)=0
$$

It now becomes evident that the integrating factor is $\partial(0) / \partial x_{i}$. Multiplying by $i t$, summing over $i$ and using (5-9) leads to

$$
\frac{d}{d t}\left|\frac{B_{i}}{\rho} \frac{\partial x_{i}^{(0)}}{\partial x_{i}}\right|=0
$$

and since

$$
\frac{\partial x_{j}}{\partial x_{i}} \underset{t \rightarrow 0}{\longrightarrow} \delta_{i j}
$$


$(5-12)$ integrates to

$$
B{ }_{j}^{(0)}=\frac{\rho(0)}{\rho} B_{i} \frac{\partial x_{j}^{(0)}}{\partial x_{i}}
$$

Usually, ${ }^{(0)}$ is given and $B_{i}$ required; using eq. $(5-9)$ then gives Cauchy's celebrated integral (Cauchy, 1815)

$$
B_{i}=\frac{\rho}{\rho(0)} B_{j}^{(0)} \frac{\partial x_{i}}{\partial(0)}
$$

To investigate the form of eq. $(5-14)$ in curvilinear coordinates we adopt tensor notion and indicate contravariant components of vectors by upper indices.

Equation (5-14), in cartesian coordinates, is then written

$$
B^{i}=\frac{\rho}{\rho(0)} B^{(0) j} \frac{\partial x^{i}}{\partial x}(0) j
$$

Let this be transformed into curvilinear coordinates $y^{i}$. The transformation of $\partial x^{i} / \partial x^{(0) j}$ is obtained from the chain rule of differentiation

$$
\frac{\partial x^{i}}{\partial x^{(0) j}}=\frac{\partial x^{i}}{\partial y^{k}} \frac{\partial y^{k}}{\partial y(0) 1} \frac{\partial y^{(0) 1}}{\partial x^{(0) j}}
$$

while $B^{i}$ and $B^{(0) j}$ transform as contravariant vector components. Substituting into $(5-15)$ and using (5-9), which is valid for the 
$y^{i}$ as well, yields an equation having the same form as (5-15). That equation is therefore valid for all $x^{i}$, rectilinear and curvilinear. In particular, in orthogonal curvilinear coordinates with scale factors $h_{i}$ (e.g. Morse $\star$ Feshbach, 1953, §1.3) the proper components $B_{(i)}$ of $\underline{B}$ (i.e. those defined in terms of unit vectors) satisfy

$$
B_{(i)}=\frac{\rho}{\rho^{(0)}} h_{i}\left(\begin{array}{l}
B_{(j)}^{(0)} \\
h_{j}^{(0)}
\end{array}\right) \frac{\partial x^{i}}{\partial x^{(0) j}}
$$

(summation on $\mathrm{j}$ on $\mathrm{ly}$ )

Cauchy's integral is frequently written

$$
\underline{B}=\frac{\rho}{\rho(0)} \underline{B}^{(0)} \cdot \nabla^{(0)} \underline{x}
$$

with similar notation in the equations leading to it. The invariance of eq. (5-15) under coordinate transformation shows, however, that $\nabla^{(0)} \underline{x}$ does not transform in the manner of a second order tensor such as $\nabla_{\underline{v}}$ LSuch a transformation was assumed by Elsasser (1956), leading to the incorrect eq. (7.12) in his work]. Strictly speaking, $\nabla^{(0)} \underline{x}$ is a donble tensor field (Michal 1927, 1947; Truesdel1 and Toupin, 1960; Ericksen, 1960), the components of which may depend on two points in space - here $\underline{x}$ and $\underline{x}^{(0)}$ - and the transformation of which may simultaneously involve the local vector bases at both points. In the transformation of $\nabla^{(0)} \underline{x}$, for instance, $\nabla^{(0)}$ transforms at $\underline{x}^{(0)}$ while $\underline{x}$ transforms at the particle's given location; this is expressed 
by eq. (5-16). For a more rigorous and comprehensive treatment of double tensor fields and their applications the reader is referred to the works of Truesdell and Toupin (1960) and of Ericksen (1960)。

Canchy's integral may be generalized for finite conductivity (Maursberger, 1964)。 Let $\underline{E}^{*}$ be the electric field in a frame moving with the fluid, which to first order in $v / c$ equals

$$
\underline{E} * \underline{E}+\stackrel{l}{(v \times} \times \underline{B})
$$

Then $(4-1)$ becomes

$$
\frac{\partial \underline{B}}{\partial t}-\nabla \times(\underline{v} \times \underline{B})=-(\nabla \times \underline{E} *)
$$

Retracing previous calculations, one obtains

$$
\frac{\mathrm{d}}{\mathrm{dt}}\left(\frac{\mathrm{B}}{\rho}\right)+-\frac{\underline{\mathrm{B}}}{\rho} \cdot \nabla \underline{\mathrm{V}}=-\frac{1}{\rho}\left(\nabla \times \underline{E}^{*}\right)
$$

and with the same integrating factor as before this gives

$$
\frac{d}{d t}\left(\frac{\underline{B} \cdot \nabla \underline{x}(0)}{\rho}\right)=-\frac{1}{\rho}\left(\nabla \times \underline{E}^{*}\right) \cdot \nabla \underline{x}^{(0)}
$$

Integration then results in

$$
\underline{B}(\underline{x})=\frac{\rho}{\rho(0)}\left\{\underline{B}^{(0)}-\int_{0}^{t} J\left(\nabla \times \underline{E}^{*}\right) \cdot \nabla \underline{x}{ }^{(0)} d t\right\} \cdot \nabla^{(0)} \underline{x}
$$


where the integration extends along the track of that particle which has position vector $\underline{x}$ at time $t$. The Jacobian $\mathrm{J}$ is defined by eq. $(5-7)$ and may be expressed in terms $\nabla(0)_{\underline{x}}$ (compare Truesdel1 1954, eq. 84-1), which is done in Malersberger's work. Mauersberger also derives the magnetic analog of Ertel's vorticity formula and some applications; for more details, the reader is referred to his article.

\section{(6) Application of Cauchy's Integral}

Let $d \underline{x}$ be the (time dependent) element of length occupied by an infinitesimal fluid filament. If at $t=0$ the filament is aligned with the magnetic field, one may write

$$
d \underline{x}^{(0)}=\underline{B}^{(0)} \mathrm{d} \lambda
$$

with $d \lambda$ an infinitesimal constant. Using the chain rule of differentiation on (5-6) and the notation of (5-18) gives, for $t>0$

$$
d \underline{x}=d \underline{x}^{(0)} \cdot \nabla^{(0)} \underline{x}=\frac{\rho^{(0)}}{\rho} \underline{B} d \lambda
$$

The above implies that the motion is line-preserving as could, of course, be directly inferred from (4-8). Conversely, Cauchy's integral may be easily derived from the line-preservation and flux-preservation properties of $\underline{B}$ which follow from $(1-2)$ (Lundquist, 1951, 1952; result is expressed there in terms of

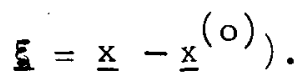

Eliminatind $d \lambda$ between the absolute values of $(6-1)$ and $(6-2)$ yields 


$$
\frac{B}{B^{(0)}}=\frac{d x}{d x} \frac{p}{\rho^{(0)}}
$$

from which the intuitive interpretation of Cauchy's integral may be summed up in three statements:

(1) The motion is line preserving.

(2) The magnetic field intensity is amplified in direct proportion to the stretching $\mathrm{dx} / \mathrm{dx}{ }^{(0)}$ of the fluid along the field's direction.

(3) The magnetic field is amplified in proportion to the bulk compression $p / \rho^{(0)}$.

Most qualitative discussions of phenomena involving motion of lines of force are based on one or more of the foregoing. Consider the differential rotation of the sun: if initially the 1 ines of force of the solar magnetic field lie in meridional planes, then the differential rotation of the solar sirface. becalse of line preservation, would tend to wind them into a pair of spirals having opposite sense in opposite hemispheres. The same process stretches solar matter along lines of force and may therefore be expected to amplify the field. This is roughly the basis of Babcock's theory of the solar cycle (1961); the energy required for the field's amplification is "ltimately supplied by the mechanism which maintains the differential rotation.

Again, when a star condenses out of a large volume of tenrons conducting gas permeated by a weak magnetic field, the field is likely to undergo considerable amplification due to 
compression. Since the field's final strength maybe high enough to contribute appreciably to the forces acting on the gas, the process is important in theories of stellar evolution. (Hoyle, 1957)

Equations (6-2) and (6-3) may also be used numerically. By following a small fluid element from a point at which $\underline{B}$ is known and noting the variation of its density and its dimension along the field, $\underline{B}$ at other points along the element's motion can be found.

Finally, situations exist in which eq. (5-18) may be used analytically. Consider, for instance, the solar wind emanating from the rotating sun. It can be assumed (Stern 1964) that near the sun the lines of force are radial (If one starts from a rigidly rotating sun problems of continuity arise). Assuming that the flow velocity $\underline{v}$ is radial and that both it and the angular velocity $\omega$ of the field's source are constant, the contravariant components of eq. (5-6), in spherical coordinates corotating with the sun, are

$$
\begin{aligned}
& r=r^{(0)}+v t \\
& \theta=\theta^{(0)} \\
& \phi=\phi^{(0)}-\Omega t=\phi^{(0)}-\frac{\omega}{v}\left(r-r^{(0)}\right)
\end{aligned}
$$

Assuming also

$$
\frac{\rho}{\rho(0)}=\left(\frac{r(0)}{r}\right)^{2}
$$


equation $(5-17)$ gives

$$
\begin{aligned}
& B_{r}=\left(\frac{r(0)}{r}\right)^{2} \quad \begin{array}{l}
(0) \\
B_{r}
\end{array} \\
& B_{\theta}=0 \\
& B_{\phi}=\left(\frac{r(0)}{r}\right)^{2} r \sin \theta \frac{B}{r} \cdot\left(\frac{\varphi}{v}\right)=B_{r} \frac{\omega r \sin \theta}{v}
\end{aligned}
$$

leading to the well-known angle (Parker, 1958)

$$
x=\operatorname{arctg}(\omega r \sin \theta / v)
$$

between the radial direction and that of $\underline{B}$. Another example of this type, an application of Cauchy's integral to a problem in dynamo theory, has been given by Parker (1955)。

\section{(7) Neutral Points in the Magnetic Field}

We now consider the case in which the conductivity $\sigma$ is finite, though still very large. When the velocity $\underline{v}$ is negligible, eq. $(4-2)$ redices to

$$
\frac{\partial \underline{B}}{\partial t}=\frac{1}{\mu_{0} \sigma} \nabla^{2} \underline{B}
$$

which yields a parabolic diffusion equation for each of the cartesian components of $\underline{B}$. The field then tends to decay exponentially with a decay time of order $\tau=\mu_{0} \sigma L^{2}$, where $L$ is a typical dimension of the field; the decay of the flux passing a 
given volume can also be visualized as a diffusive spreading-out of the lines of force with velocity of order $L / \tau=\left(\mu_{0} \sigma L\right)^{-1}$. An example of this is the decay of the magnetic field die to a current distribution in a conducting sphere (Elsasser, 1956).

The foregoing suggests that the general solution of $(4-2)$ is a blend of convection of the field by the fluid's velocity $\underline{v}$ and its diffusive decay, the relative importance of the two effects being determined by $R_{m}$. A nseful concept in this case, introduced by Sweet (1950), is the slippage velocity of lines of force. Sweet visualized the velocity of 1 ines of force in a conducting fluid as consisting of two components: a convection with the fluid's velocity $\underline{v}$ and a slippage $\underline{w}$ due to finite conductivity. In addition to finite conductivity he also considered the terms added to $\underline{E}^{\text {* }}$ in Ohm's law for dense plasmas LSpitzer, 1962 eq. $2-21$; the largest of these terms appear in eq. (1-5)] lumping

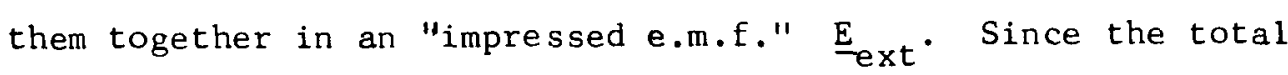
velocity of the lines of force has to satisfy $(1-2)$, one obtains

$$
\nabla \times(\underline{w} \times \underline{B})+\underline{E}_{\text {ext }}=\frac{1}{\mu_{0} \sigma} \nabla^{2} \underline{B}
$$

The component of $w$ parallel to the field is undefined in $(7-2)$ and it is natural to add

$$
(\underline{w} \cdot \underline{B})=0
$$

In what follows $\underline{E}_{\text {ext }}$ will be neglected. Removing the curl and isolating $\underline{w}$ then gives 


$$
\underline{w}=\frac{\underline{B} \times(\nabla \times \underline{B})}{\mu_{0} \sigma^{2}}+\underline{w}^{\prime}
$$

where $\underline{w}^{\prime}$ is a relabeling velocity satisfying eq. $(3-4)$. Disregarding $\underline{w}^{\prime}$, the slippage velocity is proportional to the magnetic body force acting on the fluid. One thus gets the picture of a viscous interaction between the fluid and the lines of force, with the lines exerting a force on the fluid proportional to their relative velocity. The order of magnitude of $\underline{w}$ is $\left(\mu_{\circ} \sigma \mathrm{L}\right)^{-1}$ and it thus furnishes a more precise definition of the "diffusion velocity" previously introduced.

The analogy with viscosity can be expressed in a more quantitative manner. It may be shown (for details and applications, see Batchelor, 1950, and Landau \& Lifshitz, 1960, §55) that in an incompressible fluid with viscosity $v$, subject to gravity and pressure, equation $(1-1)$ for the vorticity $\Omega$ is replaced by

$$
\frac{\partial \Omega}{\partial \mathrm{t}}-\nabla \times(\underline{\mathrm{v}} \times \underline{\Omega})=v \nabla^{2} \Omega
$$

which formally resembles $(4-2)$ 。 Because of this analogy, the quantity

$$
v_{m}=\left(\mu_{0} \sigma\right)^{-1}
$$

is sometimes called the magnetic viscosity (E1sasser, 1956, eq. 2.13).

Equation $(4-2)$ is of prime importance in the theory of self-sustaining fluid dynamos. Special cases have been solved by E1sasser (1946a, 1946b, 1947), Parker and Krook (1956), 
Stern (1964) and others, but the general treatment is difficult; it does not reduce to scalar diffusion equations in the convected frame for $\alpha$ and $B$. This is in keeping with the general rule that, except for simple cases, equations involving the vector Laplacian do not reduce to a set of corresponding equations involving scalar Laplacians.

In astrophysical applications many cases occur in which $\sigma$ is exceedingly large. It has been pointed out by Sweet (1956) and later by Parker (1957) and Pestschek (1963) that in the se cases, if the magnetic field possesses neutral points at which its intensity vanishes, dissipative effects can be greatly accelerated in the vicinity of these points. Following these anthors, we now investigate highly conductive fluids in which the effects of finite conductivity are small everywhere except in the neighborhood of nettral points.

Astrophysical interest in nentral points dates from an observation by Giovanelli (1947) that solar flares occur preferentially in the vicinity of neutral points of sunspot fields. This idea - which still lacks complete confirmation because of the difficulty in identifying neutral points on the sun - led Dingey (1953, 1958, 1963) to develop a theory of acceleration of charged particles by electric fields near neutral points. Much of what is said here about the classification and behavior of neutral points follows Dungey's work.

The magnetic field $\underline{B}$ near a neltral point $\mathbf{N}$ may be expressed, to lowest order, by a Taylor expansion 


$$
\underline{B}=\underline{a} \cdot \underline{r}
$$

where $\underline{r}$ is the radius vector to $N$ and $\underline{a}^{T}=\left.\nabla \underline{B}\right|_{N}$ (superscript $T$ denotes transpose) is a constant dyadic with zero trace, symmetric if the current density at $\mathrm{N}$ vanishes. The direction of $\underline{B}$ at $\mathrm{N}$ is undefined; nevertheless, it is useful to define a "line of force through $\mathrm{N}^{\prime}$ as a continuors smooth line passing $\mathrm{N}$ and tangent to $\underline{\mathrm{B}}$ at all other points (the term "neutral line" is sometimes used, but this is apt to be confused with a line along which $\underline{B}$ vanishes)。

Neutral points can be classified (Dungey 1953) by the number of lines of force passing them. Let such a line have a unit tangent $₹$ at $N$. At points on the line infinitesimally close to $\mathrm{N}$

$$
\begin{aligned}
& \underline{\mathbf{r}}=\lambda \underline{\xi} \\
& \underline{\mathrm{B}}=\lambda \underline{\mathrm{a}} \cdot \underline{\xi}=\mu(\lambda) \underline{\xi}
\end{aligned}
$$

the last equality following from the condition that $\underline{B}$ is tangent to the line. Since $\lambda$ and $\mu$ are both real (though infinitesimal), $\xi$ must be an eigenvector of a corresponding to a real eigenvalue. A line though $\mathrm{N}$ on which $\underline{\mathrm{B}}$ vanishes is also considered here a line of force, corresponding to an eigenvalue zero. A bundle of lines of force having a common tangent at $\mathrm{N}$ is cornted as one line only.

There exist therefore two kinds of neutral points. If a has only one real eigenvalue, there is only one line of force passing 
through $\mathrm{N}$, which is then called (by the shape of lines of force in its vicinity) an o-type neutral point. The behavior of $\underline{B}$ near an O-type neutral point forms the basis of Cowling's theorem (Cowling, 1934) on the impossibility of stationary axisymmetric fluid dynamos. Alternatively, there may exist 3 real eigenvalues $\lambda_{i}$, with corresponding unit vectors $\Sigma_{i}$, in which case 3 lines of force or bundles of lines pass through

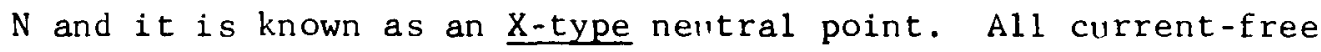
nentral points belong to this class, for $\underline{a}$ is then symmetric and has only real eigenvalues. In what follows, only $X$-type neutral points will be considered.

Becalrse the divergence of $\underline{B}$ vanishes, the three eigenvalues add up to zero and cannot all be of the same sign. It will be assumed here that $\lambda_{1}$ and $\lambda_{2}$ are positive and $\lambda_{3}$ negative; the alternate case of two negative eigenvalues is then obtained by reversing the magnetic field everywhere, a procedire which, it will be noted, does not change the disposition of the magnetic force. The vectors $\bar{\varepsilon}_{i}$ will be chosen of unit length and their sense is determined by stipilating that the scalar products $\left(\xi_{1} \cdot \xi_{2}\right),\left(\xi_{2} \cdot \xi_{3}\right)$ and $\left(\xi_{1} \xi_{2} \xi_{3}\right)$ are positive [the sign of (E. $\left.\Sigma_{3}\right)$ cannot be freely chosen].

If $\underline{r}$ lies in the plane of $\xi_{1}$ and $\xi_{2}, \underline{B}$ is contained in that plane as well. Choosing an orthogonal cartesian system for which this is the $(x, y)$ plane, it is found that everywhere in this plane the quadratic form

$$
\underline{r} \cdot \underline{B}=\underline{a}: \underline{r} \underline{r}
$$


involves only the top left (2x2) submatrix a' of a, which has the positive eigenvalues $\lambda_{1}$ and $\lambda_{z_{2}}$. If in addition no current flows in the $z$-direction, $\underline{a}^{\prime}$ is symmetric as well and therefore positive definite. Then 21 and $\underline{2}$ are orthoronal, $\underline{\underline{E}} \underline{\mathrm{B}}$ is positive for all directions and the lines of force point ontwards everywhere in the $\left(\xi_{1}, \xi_{2}\right)$ plane. In this case each of the lines of force to which $\xi_{1}$ and $\xi_{2}$ are tangent spreads out into a bundle of outward-going lines, the two bundles, between them, covering the plane. In the general case in which $\xi_{1}$ and $\xi_{2}$ are inclinced at some angle $\theta, \underline{a}^{\prime}$ is positive definite only if

$$
\cos ^{2} \theta<4 \lambda_{1} \lambda_{2} /\left(\lambda_{1}+\lambda_{2}\right)^{2}
$$

In other cases the lines of force diverging fron $\mathrm{N}$ bend so as to approach $\mathrm{N}$ again before finally heading away (compare Dingey, 1963).

The claim is often made that lines of force are broken and reconnected at neutral points. This will now be examined.

In the approximation (7-5) the cartesian components of $\underline{B}$ are linear combinations of those of $\underline{r}$ and therefore, in this order of approximation, $\nabla^{2} \underline{B}$ vanishes. Neglecting $\underline{E}$ ext in $(7-2)$, the slippage velocity $\underline{w}$ then satisfies $(3-4)$ and therefore has the character of a "relabeling velocity", by which lines of force in a given pattern are transformed among themselves, without change to the pattern as a whole.

$$
\text { Assume for simplicity a field in which there is no flirid }
$$


motion: in such a field, $\underline{w}$ will be the entire velocity of field lines. Near the nelltral point, $\underline{w}$ is large (it diverges at $N$ ) and it transforms field lines within a fixed pattern, but at large distances fron $\mathrm{N}$ it diminishes rapidly to a negligible magnitude.

Consider a line of force which initially passes points $A B C$ near $\mathrm{N}$ (Figure 3). A time interval dt later the 1 ine will have shifted a distance $\underline{w} d t$. At the "roots" of the line far from $N$ this shift is negligible, so that if one defines a line by its roots one will be still observing essentially the same line. Near $N$, however, there will be a real change, with the points $A B C$ moving to $D E F$ and the line becoming differently connected.

The slippage velocity becomes infinite at $N$. This is necessary to enable point $A$ to "turn a corner" at $N$ and transform to $D$ while keeping up with $B$ and $C$ as they transform smoothly into $\mathrm{E}$ and $\mathrm{F}$. As a consequence, no matter what the flow velocity $\mathrm{v}$ of the condrcting fluid is, there will always exist a small neighborhood of $\mathrm{N}$ in which the convection of field lines by the fluid velocity can be neglected and their velocity be taken as $\underline{w}$.

To obtain some more insight into the slippage process near neutral points we introduce an essentially two-dimensional model, with vectors $\xi_{1}$ and $\xi_{2}$ making an angle $\theta$ in the $(x, y)$ plane and $\xi_{3}$ following the $z$-axis, and with corresponding eigenvalues $\lambda,-\lambda$ and 0 . The diffusion velocity $\underline{w}$ along the direction of $\left(\xi_{1}+\xi_{2}\right)$ is then radial (this corresponds to diffusion fron $N$ to $D$ in Figure 3) and its magnitude at distance $x$ is 


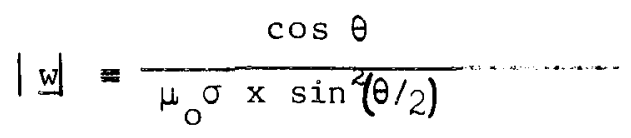

One notes that the magnitude of $\underline{B}$, represented by $\lambda$, is absent and that $\underline{w}$ increases as the angle between the axes diminishes (it is zero for $\theta=\pi / 2$ becalnse then $\nabla \times \underline{B}$ vanishes) and also is inversely proportional to the distance from $N$. (8) The Evolution of Neutral Points

The volume force on the fluid ( $T$ denotes transpose) is

$$
\underline{F}=\underline{j} \times \underline{B}=\frac{1}{\mu_{0}}\left(\underline{a}-\underline{a}^{T}\right) \cdot \underline{a} \cdot \underline{r}
$$

The force vanishes at the neutral point and, because of its orthogonality to $\underline{B}$, has no radial component on the main axes defined by the $\underline{\xi}_{i}$ a In general, the radial component of $\underline{F}$ at a point

$$
\underline{r}=\Sigma u_{i} \underline{\xi}_{i}
$$

is given by

$$
r F_{r}=\frac{1}{2} \Sigma\left(\underline{\xi}_{i} \underline{\xi}_{j}\right) u_{i} u_{j}\left(\lambda_{i}-\lambda_{j}\right)^{2}
$$

This component vanishes on the quadric surface obtained by equating (8-2) to zero, which is evidently a cone, since it contains three non-coplanar straight lines (the main axes) intersecting at the origin. The force then pushes inward inside the cone and away fron $\mathrm{N}$ ontside it - or vice versa, 
depending on the sign of $\left(\underline{\xi}_{1} \cdot \underline{\xi}_{3}\right)$. If the fluid flow follows this force, it will tend either to open the cone up into the vicinity of a plane ("the neltral plane") or to close it into a line ("neutral filament"), either of them passing through $\mathrm{N}$.

Such "folding" of the field was obtained by Dingey (1953, 1958) when he first treated the fluid's motion near the nertral point. His calculation assumed that pressure and conductivity can be neglected and that the fluid velocity vanishes at the nentral point, so that it can be expanded, analogously to $(7-5)$

$$
\underline{v}=\underline{b} \cdot \underline{r}
$$

Equation (1-2) then gives a (dyadic) equation

$$
\frac{\partial \underline{a}}{\partial t}=(\underline{b} \cdot \underline{a})-(\underline{a} \cdot \underline{b})-\underline{a} \Sigma b_{i i}
$$

(forming the scalar product with $\underline{\underline{r}}$ recovers $1-2$ ). Similarly, the force equation

$$
\frac{\partial \underline{v}}{\partial t}+\underline{v} \cdot \nabla \underline{v}=(\underline{j} \times \underline{B})
$$

redices to

$$
\frac{\partial \underline{b}}{\partial t}+(\underline{b} \cdot \underline{b})=\frac{1}{\mu_{0}}\left(\underline{a}-\underline{a}^{\tau}\right) \cdot \underline{a}
$$

If initially both $\underline{v}$ and $\underline{j}$ vanish, so that the magnetic axes are orthogonal and $\underline{a}$ is symmetric, Dungey showed that a small pertirbation 
amplifies itself and produces a flow diminishing the angle between axes of oppositely signed eigenvalues (angle $\theta$ in Figure 3). The current density j meanwhile increases, until the electric field $\underline{E}^{*}$ (in the fluid's frame) becomes appreciable. Dungey called this an "instability"; it was his idea that such a development takes place in solar flares and that the current then becomes a "discharge" accelerating particles to high energies. While Dingey's derivation is formally correct, cantion must be exercised in its interpretation, for the growth of a local instabil. ity associated with $N$ contradicts the existence of stable neutral points. Such points do exist. Consider for instance two fixed parallel magnetic dipoles, directed along the line connecting them and immersed in an infinite homogeneons conducting fluid at rest (Figure 4). In such a configuration a neutral point (more precisely, a circular locns of neltral points) is formed between the dipoles, resembling in all respects the one investigated by Dungey . If this system were unstable, one wonld still expect that after all fluid motions and volume currents had decayed there would remain a homogeneous medium with two embedded dipoles. The final energy then equals the initial one and there is none left to drive the instability.

To understand Dungey's results one notes that the effect dediced by him is not confined to the neighborhood of $\mathrm{N}$. Indeed, the folding of the main axes and of the associated system of field lines extends as far as the approximation (7-5) does, and it is 
not at all certain that some surface can be found, enclosing a relatively small region around $\mathrm{N}$, beyond which the perturbation is negligible. In the example given, at least, it seems that no such surface exists and that the "folding" near $N$ is only part of a deformation involving the entire field, becalse the only source of energy available here is the relative potential energy of the two dipoles. In particular, allowing the dipoles to attract each other (Jackson 1962, \$4.2) through a finite distance will release energy and will also fold the lines near the neutral point in the manner described by Dingey. It thus seems that Dingey's mechanism is actually the description of a lack of equilibrium in the entire field rather than of an instability associated with the nentral points.

A problem somewhat similar to Dingey's was treated by Chapman and Kendal1 (1963) who considered a line along which the field vanishes, surrounded by a tube of conducting fluid with finite cross section. In this case Dungey's instability proceeds without reference to the magnetic sources, since the fluid is decoupled from them by the vacuum surrounding the tube. Such decoupling seems unlikely, however, in astrophysical applications.

To obtain any local behavior at the neutral point while the field is held fixed at some distance from it, one has to extend the expansion (7-5) at least to second order. In principle, equations $(8-4)$ and $(8-6)$, generalized to this order, or the "1se of an energy principle, world then show under what conditions, if 
any, can a purely local instability proceed at the neutral point. Attention was first called to the importance of motion of the field's somrces by Sweet $(1956,1958)$. He considered in particnlar the neutral point between the two dipoles described previonsly (Figure 4) and assumed each dipole to be embedded in a mass of condrcting fluid, the two masses moving in opposite directions towards the nentral point.

Sweet did not restrict the model to electromagnetic forces alone. In this connection, he noted that forces due to pressure gradients will predominate near the nentral point, since the electromagnetic force tends there to zero. The flow prodiced by mechanical forces when two fluid masses are pressed together is similar to the one occurring in Dungey's model: the fluid is squeezed out along a plane or line. If the nentral point is initially on such a "nentral plane" (or possibly, "nevtral filament Sweet expected the flow to distort the magnetic field as was pre viously described, leading to its flattening near the plane and to the brild wp of crrrent density and electromagnetic forces. The process is finally limited by the necessity of maintaining a narrow region by which the fluid may escape from the neighborhood of the neutral point. It is in this final state that enhanced diffusion near the nentral point takes place.

The following simple model is due to Parker, who followed Swee ideas quantitatively in considerable detail (1957, 1963)。Parker assumed that the region around the neutral point $\mathrm{N}$ collapses into a thin "boundary layer" of constant thickness $2 \delta$. The fluid 
approaches the layer with velocity u along the direction

normal to it, which will be chosen as the $\mathrm{x}$ axis; the $\mathrm{y}$ direction, along which the fluid is squeezed ont, is then that of the field ${ }_{\text {B }}$ far from the layer, which is assumed to be of constant magnitude but having opposite polarities on different sides of the layer. The magnetic pressure on the flanks of the boundary layer is balanced, near $\mathrm{N}$, ty an increase in fluid pressure.

Let the layer have a finite length $2 \mathrm{~L}$ in the $\mathrm{y}$ direction. At $|y|=L$, the fluid leaves the layer and if the field and pressure have dropped close to zero near this point, the fluid's kinetic energy density $\rho v^{2} / 2$ will be roughly that acquired by a pressure drop of $\mathrm{B}_{0}^{2} / 2 \mu_{0}$. This means, then, that the velocity of the fluid leaving the layer is of the order of the Alfvén velocity

$$
v_{A}=\frac{B_{0}}{\sqrt{\mu_{0} \rho}}
$$

By continuty

$$
\mathrm{V}_{\mathrm{A}} \delta=\mathrm{uL}_{\mathrm{L}}
$$

and if $u$ and $L$ are given, $\delta$ may be found.

Inside the layer opposing field lines diffuse towards each other with a velocity of the order $\left(\mu_{0} \sigma \delta\right)^{-1}$ and ultimately merge. Ontside the boundary, field lines move with the fluid's velocity $u$. In order for the two motions to be continuous, one needs 


$$
u \approx\left(\mu_{0} \sigma \delta\right)^{-1}
$$

Eliminating $\delta$ between the last two eqrations gives

$$
u \approx\left(V_{A} / \mu_{0} \sigma L\right)^{\frac{1}{2}}
$$

In applying the above to the sun Parker assumed that a solar flare arises when two regions containing opposing magnetic fields are pushed against each other with velocity $u$. The field intensity, density and the total energy released can be estimated from observations; from the total energy, the thickness $D$ of the region of field annihilation is found to be of the order of $10^{4} \mathrm{~km}_{\star}$ The total time $\mathrm{D} / \mathrm{u}$ for the annihilation of magnetic energy is then $10^{4}-10^{6}$ seconds, as compared to observed times of order $10^{2}$ seconds

The above argument may be modified taking into account more details of the field's structure near the nentral point, assiming that it has the form given in Figure 3 with the angle $\theta$ small but finite. There will exist a region around $\mathrm{N}$ in which $\underline{w}$ predominates, and as a simple approximation one may neglect $\underline{v}$ in this region and neglect $\underline{w}$ ortside it. If $\delta$ is the extent of the region in" $x$ direction (along the line NA in Figmre 3) then its length in the $y$ direction will be of the order $\delta / \sin z \theta$.

Equation (8-9) then expresses the fact that on the boindary of this central region, on the $x$-axis, the slippage velocity equals 
the impinging fluid velocity. However, since this transition occurs close to a stagnation point of the flow, the impinging velocity may be much less than the velocity "of the fluid far from the layer. Writing the impinging velocity as $€ u$, where $\epsilon<1$, one finds that a factor $\epsilon^{-\frac{1}{2}}$ must be added to equation (8-10). If $\varepsilon$ is very small, this increases $\boldsymbol{u}$ considerably and achieves a corresponding reduction in annihilation time.

In addition, unless the angle $\theta$ is extremely small, the central region will occupy only part of the boundary layer, terminating at about $|y|=\delta / \sin \theta$. Beyond this point the layer is likely to broaden; it will certainly do so if the flow resembles that given by equation $(8-3)$. Equation (8-8) then also requires modification and again this leads to an increase in ".

A more elaborate extension of Parker's model was postillated by Petscheck (1963), who divided the boundary layer into two regions. In a section $0<|y|<y^{*}$ straddling the neutral point the mechanism suggested by Sweet and Parker holds; for $y *<|y|<L$, however, the layer diverges at an angle of abont 0.1 radians, the fluid velocity is maintained near $\mathrm{V}_{\mathrm{A}}$ and magnetic energy is removed by propagation of hydromagnetic waves. In application to solar flares, times of the order of 100 seconds are obtained, because the length $2 \mathrm{y}$ * of the central region is now much less than the total length $2 \mathrm{~L}$ and therefore the thickness 20 near $\mathrm{N}$ is correspondingly reduced.

Petschek also derives an upper limit to the rate of line re- 
combination. The main disadvantages of his approach are that it is not clear that the "wave region" envisioned will actrally be created and that his assumption of a curl-free magnetic field everywhere ontside the boundary layer leads to the correct maximum recombination rate.

In conclusion it should be stressed that the theory of nentral points is very far from complete at this stage. Almost all existing theories assume two-dimensional structure, the achievement of a nearly time-independent collapsed state and the stagnation of the fluid flow at the nentral point, any or all of which may not be justified. Even if an equilibrium in the collapsed state occurs, it may be unstable (Parker, 1963; Jaggi, 1963; Severny, 1963)。Whether such instability merely leads to small-scale turbulence and to an increase in effective resistivity or actually disrupts the structure of the boundary layer remains yet to be seen.

The most important deficiency of the theory of nertral points, however, is the lack of experimental confirmation. Though the theory was originally conceived to explain solar flares, the complexity of sinspot fields and of flares and the difficulty in observing them have prevented, so far, any conclusive proof of an association between flares and neutral points. There may be some additional evidence from the "tail" of the earth's magneto. sphere (Ness, 1965) where a region of weak magnetic field, commonly called "nentral plane", has been observed near the magnetic equator on the night side of the earth, separating oppositely directed 
fields. Both the magnetic field and particle density are much lower here than near solar flares; whether rapid reconnection of field lines (Axford et al.,1965) and particle acceleration (Speiser, 1965; Coppi et al., 1966) occur under these circumstances is still subject to controversy (Dessler, 1965).

Finally, an attempt to investigate neutral points experimentally in the laboratory has been recently started (Bratenahl * Hirsch, 1966). The nelitral point (or rather, a line of nelitral points) was obtained by allowing two expanding cylindrical fields to collide, leading to a sitration somewhat resembling that investigated theoretically by Green (Green, 1965). Observations of the evolution of these fields strongly suggest that a reconnection of magnetic field lines does in fact occur. However, instead of the field tending to a relatively slowly varying collapsed state, oscillations are observed with field lines near the neltral point alternately folding and opening.

It this appears that what is most needed in the stidy of nentral points is observational evidence, either in the laboratory or in space. Until such evidence becomes available, the preceding can serve as no more than a tentative theory.

Acknowledgment

The author thanks all those who critically reviewed drafts of this article, especially Professor E. Parker, who pointed out some errors in the original version, Dr. T. G. Northrop, Dr. R. Miller and Professor W. Elsasser. 


\section{Captions to the figures}

Figure 1 - No caption

Figure 2 - Two Interesecting Flux Tubes

Figure 3 - The Magnetic Field Lines Near an X-type Neutral Point

Figure 4 - The Neutral Point Formed Between Two Parallel Dipoles 


\section{References}

Alfvén, H., On the Affect of a Vertical Magnetic Field

in a Conducting Atmosphere, Arkiv för Matematik, Astronomi

och Fysik 29-A, No. 11, (1942).

Alfvén, H., On the existence of Electromagnetic-Hydrodynamic

Waves, Arkiv för Matematik, Astronomi och Fysik 29-B, No. 2 (1942).

Axford, W. I., Petschek, H. E. and Siscoe, G. L., Tail of the

Magnetosphere, J. Geophys. Res., 70, 1231 (1965).

Babcock, H. W., The Topology of the Sun's Magnetic Field and

the 22-year Cycle, Astrophys. J., 133, 572 (1961).

Batchelor, G. K., On the Spontaneous Magnetic Field in a Con-

ducting Liquid in Turbulent Motion, Proc. Phys. Soc. of London

A 201, 405 (1950).

Brand, Louis, Vector and Tensor Analysis, John Wiley and Sons, (1947).

Bratenahl, A. Hirsch, W., An Experimental Study of a Neutral

Point in a Plasma, Paper 66-162, AIAA Plasmadynamics Conf.,

Monterey, March 1966; also Bu11. Am. Phys. Soc., $\underline{11}$, 580 (1966).

Cauchy, A.-L., Theorie de la Propagation des ondes a la Surface

d'un Fluide Pesant d'une Profondeur Indefinie, Mem. Divers

Savants (2) 1,3 (1816); Deuvres (1) 1,5

Chapman, S. \& Kendal1, P. C., Liquid Instability and Energy

Transformation Near a Magnetic Neutral Line : A Soluble Non-linear Hydromagnetic Problem, Proc. Roy. Soc., A-271, 535 (1963). .

B. Coppi, G. Laval \& R. Pellat: Dynamics of the Geomagnetic Tail,. Phys. Rev. Let. 16, 1207 (1966).

Cowling, T. G., The Magnetic Field of Sunspots,"Mon. Not. Roy.

Astr. Soc.", 94, 39 (1934). 
Dessler, A。J., \& Michel, F. C., Magnetospheric Models, Proc.

of Symp. on Radiation Trapped in the Earth's Magnetic.Field, Bergen (1965), to be published by D. Reidel, 1966.

Dungey, J. Wo, Conditions for the Occurence of Electrical

Discharges in Astrophysical Systems, Phil. Mag. Series 7, 44, 725 (1953)

Dungey, J. W., Cosmic Electrodynamics, Cambridge Univ. Press (1958)。 Dungey, J. W., The Structure of the Exosphere, or, Adventures in Velocity Space, 'in: Geophysics, The Earth's Environment (proceedings of the 1962 Les Houches Summer School), Gordon \& Breach (1963). Elsasser, W. M., Induction Effects in Terrestial Magnetism (I - III), Phys.Rev. 69, 106 (1946); 70, 202 (19wu) 72, 821 (1947). Elsasser, W. M., Hydromagnetism : A Review, Am. J. o Phys. $\underline{23}, 590(1955) ; \underline{24}, 85(1956)$.

Elsasser, W. M., Some Dimensional Aspects of Hydromagnetic Phenomena, in Magnetohydynamics, R. K. M. Landshoff Ėit., Stanford University (1957)。

Elsasser, W. M., Hydromagnetic Dynamo Theory, Rev. of Mod. Phys。 $28,135(1956)$.

Ericksen, J. L., Tensor Fields (appendix to"The Classical Field Theories", by C. Truesdell \& R. A. Toupin.), Handbuch der Physik (S. Flügge edit.) III/1, p. $794-858$ (1960).

Enler, L., Sectio Secunda de Principiis Motus Fluidorum, Novi Commentarii Acad. Sci. Petropolitanae, 14, 270 (1769); reprinted in Leonhardi Enleri Opera Omnia, Series II, Vo1. 13, p. 73, pub. by the Swiss Soc. for Natural Sci. (1955). 
Giovanelli, R. G., Electric Phenomena Associated with Sunspots, Mon. Not. Roy. Astr. Soc., 107, 338 (1947).

Goldstein, Sidney, Lectures on Fluid Mechanics, (Lectures in Applied Mathematics - Proceedings of the Summer Seminar, Bollder, Colorado 1957, Vol. II), Intersci. Pub. (1960). Green, R. M., Modes of Annihilation and Reconnection of Magnetic Fields, in "Stellar and Solar Magnetic Fields" (R. Lust Edit.) I.A.U. Symp. No.. 22, North Holland P.ub, Co. (1965). Helmholtz, H., Über Integrale der Hydrodynamische Gleichungen, Welche den Wirbelbewegungen Entsprechen, J. Reine Angew. Math 55, 25, (1858); translated by P. G. Tait,"On Integrals of the Hydrodynamical Equations, which Express Vortex-Motion", Phil. Mag. (4) 33, $485(1867)$.

Hoyle, Fred, The Build-Up of Large Magnetic Fields Inside Stars, p. 29, Magnetohydrodynamics", R. K. M. Landshoff Edit., Stanford Univ. Press (1957).

Jackson, J. D., Classical Electrodynamics, John Wiley \& Sons (1962). Jaggi, R. K., A Mechanism for the Dissipation of the Magnetic Field in Solar Flares, AAS-NASA Symp. on the Phys, of Solar Flares (1963) p. 419 of proceedings; U. S. Gov. Print. Ofc. (1964). Lamb, Sir Horace, Hydrodynamics, First Edit. 1879; 5th Edit. repub. by Dover Pub. Landau, L. D. \& Lifshitz, E. M., Electrodynamics of Continuous Media, (Vol. 8 of "Course of Theoretical Phys.), English Translation Pergamon Press (1960). 
Lundquist, Stig, On the Stability of Magneto-Hydrostatic Fields, Phys. Rev。 83, 307 (1951).

Lundquist, Stig, Studies in Magneto-Hydrodynamics, Arkiv för Fysik 2, 297 (1952).

McDonald, K. L., Topology of Steady Current Magnetic Fields, Am. J. of Phys. 22, 586 (1954).

Maversberger, Peter, Zur Kinematik der Magnetischen Feldlinien in Plasma, Pure « Applied Geophys. 57, 143 (1964).

Maxwel1, J.C., A Treatise on Electricity and Magnetism, Oxford (1873); 3rd Edit, reprint. by Dover Pub. (1954).

Michal, Aristotle D., Fundamentals of R-Dimensional Manifolds Adinitting Continuous Groups of Transformations, Trans. Amer. Math. Soc., 29, 612 (1927).

Michal, Aristotle D., Matrix and Tensor Calculus, John Wiley \& Sons $(1947)$

Milne-Thomsan, L. M., Theoretical Hydrodynamics, The McMillan Co. $(1955)$.

Morse, P.M. Eeshbach, H., Methods of Theoretical Physics, MicGraw Hill (1953).

Ness, Norman F., The Earth's Magnetic Tail, J. Geophys. Res. 70, $2989(1965)$.

Newcomb, W. A., Motion of Magnetic Lines of Force, Annals of Phys. 3. $347(1958)$. Northrop, T. G。, Adiabatic Charged-Particle Motion, Rev. Geophys., $1,283(1963)$. 
O'Brien, V., Axi-Symmetric Magnetic Fields \& Related Problems,

J. of the Franklin Inst., 275, 24 (1963).

Parker, E. N., Hydrodynamic Dynamo Models, Astrophys. J., 122, 293 (1955).

Parker, E.N.., \&M.Krook, Diffusion \&evering of Magnetic Lines of Force, Astrophys. J., 124, 214 (1956).

Parker, E. N., Newtonian Development of the Dynamical Properties of Ionized Gases of Low Density, Phys. Rev. 107, 924 (1957).

Parker, E. N., Sweet's Mechanism for Merging Magnetic Fields in

Conducting Fluids, J. Geophys. Res., 62, 509 (1957).

Parker, E. N., Dynamics of the Interplanetary Gas and Magne tic

Fields, Astrophys. J., 128,664 (1958).

Parker, E. N., The Solar-Flare Phenomenon and the Theory of

Reconnection and Annihilation of Magnetic Fields, Astrophys. J.

Suppl., 77 (Vol. VIII, 177, 1963)

Petschek, H. E., Magnetic Field Annihilation, AAS-NASA Symp. on

the Phys. of Solar Flares, 1963; p. 425 (Pub. by U. S. Govt. Print.

Ofc. 1964).

Phillips, H. B., Vector Analysis, John Wiley \& Sons (1933).

Prim, R. «Truesde11, C., A derivation of Zorawski's Criterion for

Permanent Vector-Lines, Proceedings of the Amer. Math. Soc., 1, 32 (1950).

Ray, Ernest C., On the Motion of Charged Particles in the Geomagnetic Field, Annals of Phys., 24, 1 (1963).

Severny, A. B., On Plasma Layer Instability with the Neutral

Point of a Magnetic Field, Sov. Astro. (Astro. Zhurnal Trans1.) 
6, $770(1963)$.

Speiser, T. W., Particle Trajectories in Model Current Sheets, J. of Geophys. Res., 70, 4219 (1965).

Spitzer, J., Jr., Physics of Fully Ionized Gases, Intersci. Pub. 2nd Edit. (1962).

Stern, D. P., A Simple Model of the Interplanetary Magnetic

Field, Planetary \& Space Sci., 12, 961 (1964).

Sweet, P. A., The Effect of Turbulence on a Magnetic Field, Mon. Not. Roy. Astr. Soc., 110, 69 (1950).....

Sweet, P. A., The Nelltral Point Theory of Solar Flares in

"Electromagnetic Phenomena in Cosmical Phys." p. 123 (Proceedings of the 6th IAU Symposium 1956 Edited by B. Lehnert); Cambridge in University Press (1958).

Sweet, P. A., The Production of High Energy Particles in Solar Flares, Suppl. del Nuovo Cinento, $\underline{8}$ (Ser. 10), 1888 (1953).

Thompson, W, B., An Introduction to Elasma Physics, Pergamon Press (1962).

Truesdell, C., The Kinematics of Vorticity, Indiana Univ. Press (1954).

Truesde11, C. \& Toupin, R. A., The Classical Field Theories, Handbuch der Physik (S. Flïgge Ed.) III/1, p. 226-793 (1960). Walén, C., On the Distribution of the Solar General Magnetic Field and Remarks Concerning the Geomagnetism and The Solar Rotation, Arkiv för Matematik, Astronomi och Fysik, 33-A, No. 18 (1946).

Zorawski, $K_{\circ}$, Über die erhaltung der Wirbelbewegung, Bulletin de 1'Academie des Sciences de Cracovie, Comptes Rendıs, 1900, p. 335. 


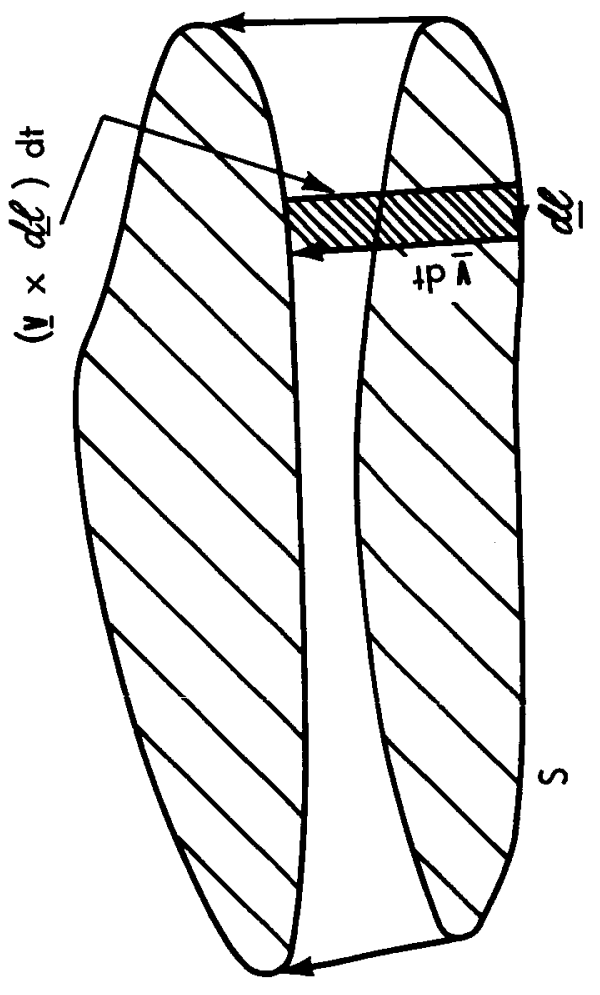

稁 


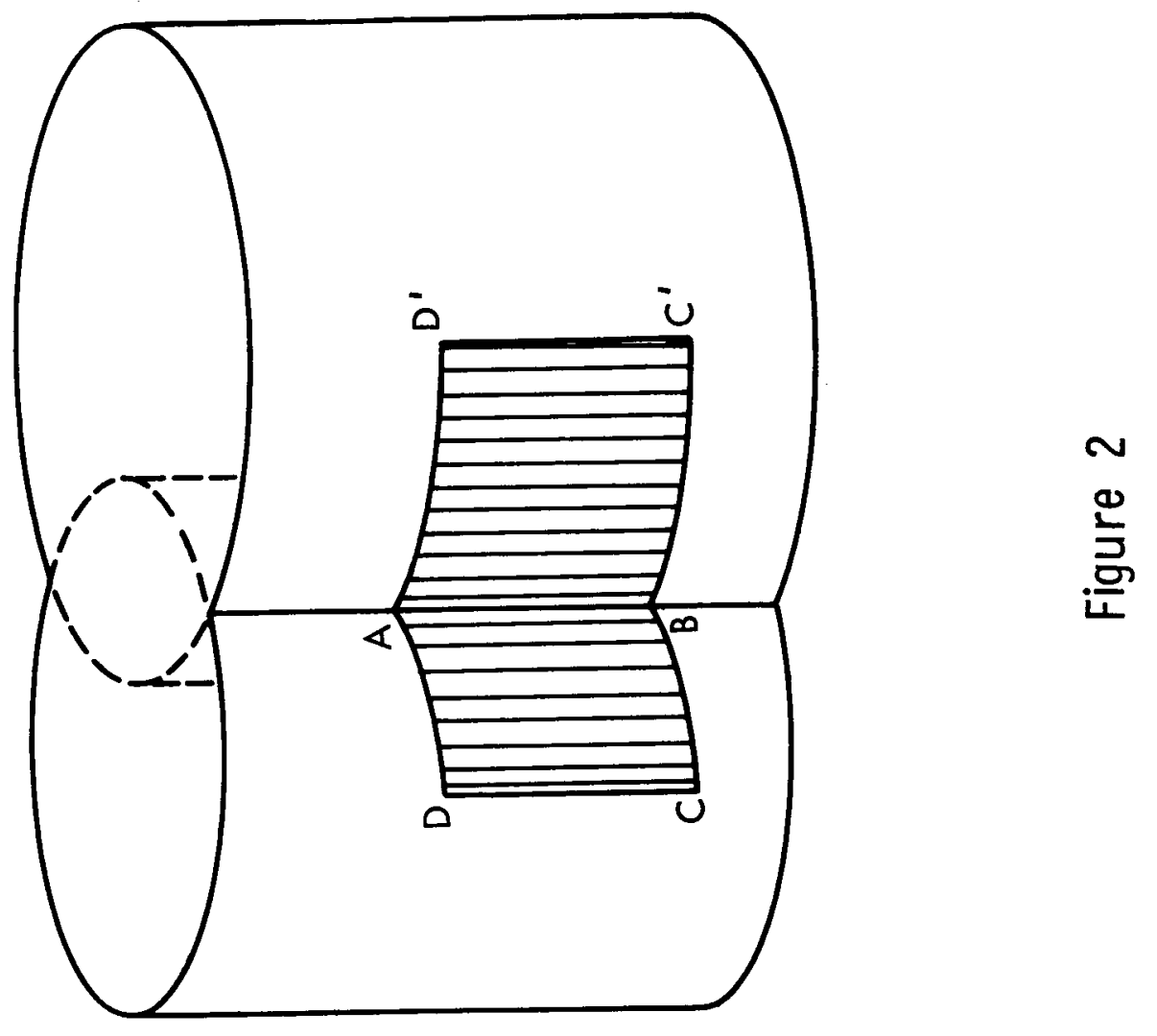




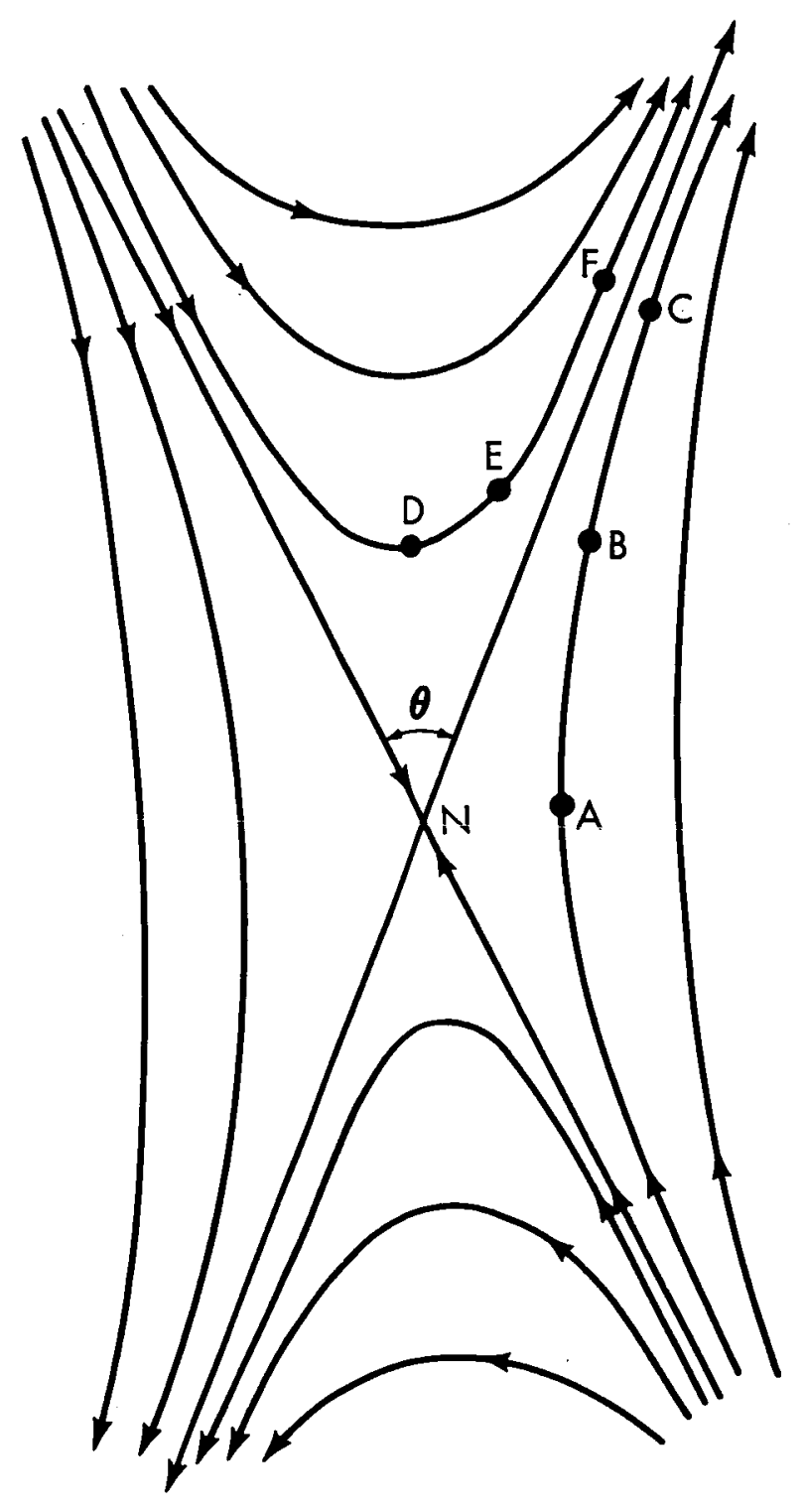

Figure 3 


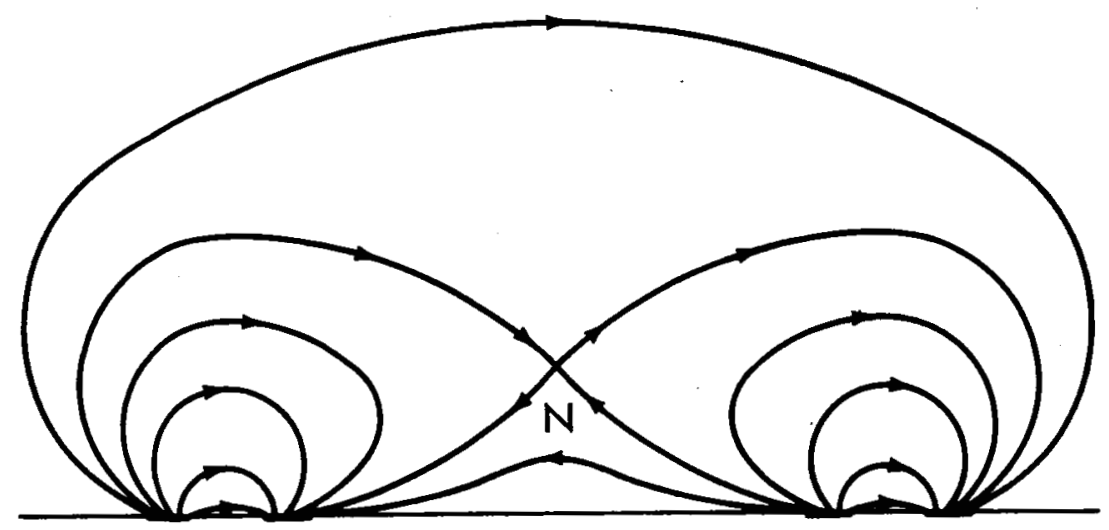

Figure 4 